\title{
Efecto antifúngico de Citrus paradisi "toronja" sobre cepas de Candida albicans aisladas de pacientes con estomatitis subprotésica
}

\author{
Antifungal Effect of Citrus paradisi "grapefruit" on strains of Candida albicans isolated from patients with \\ denture stomatitis \\ Diana Eugenia Churata-Oroyal, Donald Ramos-Perfecto², Hilda Moromi-Nakata ${ }^{2}$, Elba Martinez-Cadillo ${ }^{2}$, \\ Américo Castro-Luna ${ }^{3, a, b}$, Ruth Garcia-de-la-Guarda ${ }^{4, a, c}$
}

\section{RESUMEN}

Objetivos: Determinar la actividad antifúngica in vitro del aceite esencial de Citrus paradisi "toronja" sobre cepas de Candida albicans aislados de pacientes con diagnóstico de estomatitis subprotésica. Material y métodos: Se realizaron pruebas de sensibilidad por el método de difusión en agar con pozos. La cepa de Candida albicans se obtuvo de muestras de hisopado de mucosa de pacientes con estomatitis subprotésica y se identificó por medio de las características morfológicas de sus colonias y la prueba de tubo germinativo. El inóculo fue estandarizado al 0,5 de la escala de Mc Farland y sembrado en 12 placas con agar dextrosa sabouraud. Se hicieron pozos con un sacabocado de $6 \mathrm{~mm}$ de diámetro, se depositaron $50 \mu \mathrm{L}$ de las diferentes concentraciones del aceite esencial y se procedió a la incubación en aerobiosis a $37^{\circ} \mathrm{C}$ por 48 horas. El análisis estadístico se realizó con el programa SPSS Versión 23. Resultados: Las concentraciones al 25; 12,5; 6,25; 3,13 y 1,56 \% presentaron un halo de inhibición promedio de 12,$6 ; 10,3 ; 7,8$ y 6,8 y $6,3 \mathrm{~mm}$ respectivamente, mientras que al 0,78 y $0,39 \%$ no presentaron actividad antifúngica. La CMI promedio para Candida albicans fue de 6,25\%.v/v Se realizó la prueba de Kruskal Wallis y se determinó que existe diferencia estadísticamente significativa $\mathrm{p}<0,05$ de promedios entre las concentraciones. Conclusiones: Se determinó que el aceite esencial de Citrus paradisi "toronja", presenta actividad antifúngica sobre cepas de Candida albicans.

PALABRAS CLAVE: Citrus paradisi, Antifúngicos, Candida albicans, Estomatitis subprotética

Facultad de Odontología, Universidad Nacional Mayor de San Marcos. Lima, Perú.

Laboratorio de Microbiología, Facultad de Odontología, Universidad Nacional Mayor de San Marcos. Lima, Perú.

Facultad de Farmacia y Bioquímica, Universidad Nacional Mayor de San Marcos. Lima, Perú.

Laboratorio de Microbiología Molecular y Biotecnología, Facultad de Ciencias Biológicas, Universidad Nacional Mayor de San Marcos. Lima, Perú

Profesor ; ${ }^{b}$ Químico Farmacéutico ; ${ }^{\text {B Bióloga }}$ 


\section{SUMMARY}

Objectives: To determine the in vitro antifungal activity of essential oil of Citrus paradisi "grapefruit" on Candida albicans strains isolated from patients diagnosed with denture stomatitis. Material and methods: Sensitivity tests were performed by the agar diffusion method with wells. Candida albicans strain was obtained from swab samples of mucosa of patients with denture stomatitis and identified by morphological characteristics of colonies and germ tube test. The inoculum was standardized to $0.5 \mathrm{Mc}$ Farland scale and seeded in 12 well plates with Sabouraud dextrose agar. wells a punch of $6 \mathrm{~mm}$ diameter were made, $50 \mathrm{uL}$ of the different concentrations of essential oil were deposited and incubation proceeded to aerobically at $37^{\circ} \mathrm{C}$ for 48 hours. Statistical analysis was performed using SPSS version 23. Results: concentrations 25; 12.5; 6.25;3,13 and 1,56\% had a halo of inhibition average of $12.6 ; 10.3 ; 7.8$ and $6.8 \mathrm{~mm}$ respectively, while 0.78 and $0.39 \%$ had no antifungal activity. The MIC for Candida albicans average was $6.25 \%$.v / v Kruskal Wallis test was performed and it was determined that there is statistically significant difference $\mathrm{p}<0.05$ between concentrations of averages. Conclusions: It was determined that the essential oil of Citrus paradisi "grapefruit" presents antifungal activity on strains of Candida albicans.

\section{KEYWORDS: Citrus paradisi, antifungal agents, Candida albicans, stomatitis denture.}

\section{INTRODUCCIÓN}

Aproximadamente, el $60 \%$ de la población mundial utiliza plantas y productos derivados de ellas en su medicación, los cuales son considerados como una de las "medicinas" de gran importancia por su efectividad terapéutica (1). La medicina tradicional, en los últimos años ha cobrado importancia como una terapia alternativa al uso de medicamentos sintéticos producidos en la industria farmacéutica, los cuales producen toxicidad, recurrencia o causan resistencia, razón por la cual se está procurando investigar nuevos agentes antifúngicos más potentes y sobre todo seguros (2).

La flora peruana es muy rica en especies a las que la medicina tradicional atribuye propiedades terapéuticas muchas no investigadas (3), como es el caso de la toronja (Citrus paradisi) que pertenece al género Citrus, familia Rutaceae. Este árbol presenta un fruto de forma globular de un color amarillo claro, con un diámetro de 15 a $20 \mathrm{~cm}$, su exocarpo presenta vesículas que contienen aceites esenciales, su mesocarpio es pomposo y de un color blanco y la pulpa o endocarpio presenta tricomas con jugo blanco, rosa o rojo $(4,5)$ (Figura 1); esta planta es utilizada en muchos países como: antimicrobiano, antifúngico, antiviral, antiinflamatorio, antioxidante, astringente, también en la prevención del cáncer, regeneración celular, reducción del colesterol, mantenimiento de la salud del corazón, lupus, nefritis, artritis reumatoide, pérdida de peso, desintoxicación, conservante y limpieza (6).
Se han reportado diversas actividades del aceite esencial obtenido de las cáscaras de esta especie: actividad antifúngica frente a Candida albicans (7) y Neurospora crassa (8), actividad antibacteriana contra Staphylococcus aureus (7-9), Enterococcus faecalis y Escherichia coli $(7,9)$; además actividad larvicida $(10)$.

La cavidad bucal es una zona anatómica con importantes funciones en el organismo, constituye la mayor parte del aparato estomatognático y está sujeta a infecciones micóticas, principalmente producidas por Candida. En el ser humano esta levadura se halla formando parte de la microbiota normal de la cavidad oral (lengua, paladar y mucosa oral), tubo digestivo, vagina, tracto respiratorio y piel (11). Existen factores locales y sistémicos que favorecen su crecimiento y sobrecolonización en la mucosa oral produciendo la estomatitis (12).

Durante los últimos años se ha presentado un incremento en la incidencia de enfermedades fúngicas en seres humanos. Se considera que cuatro de cada mil pacientes que acuden a consulta dental presentan síntomas de infección candidiásica (13). En la cavidad oral las infecciones por Candida albicans adoptan varias formas clínicas, como la estomatitis subprotésica que es un proceso inflamatorio de la mucosa bucal (14), su frecuencia es del 24-60\% de los pacientes portadores de prótesis removibles y al aumentar el grado de la lesión, aumenta la presencia de la levadura, siendo la Candida albicans considerada como el microorganismo más prevalente en pacientes con 


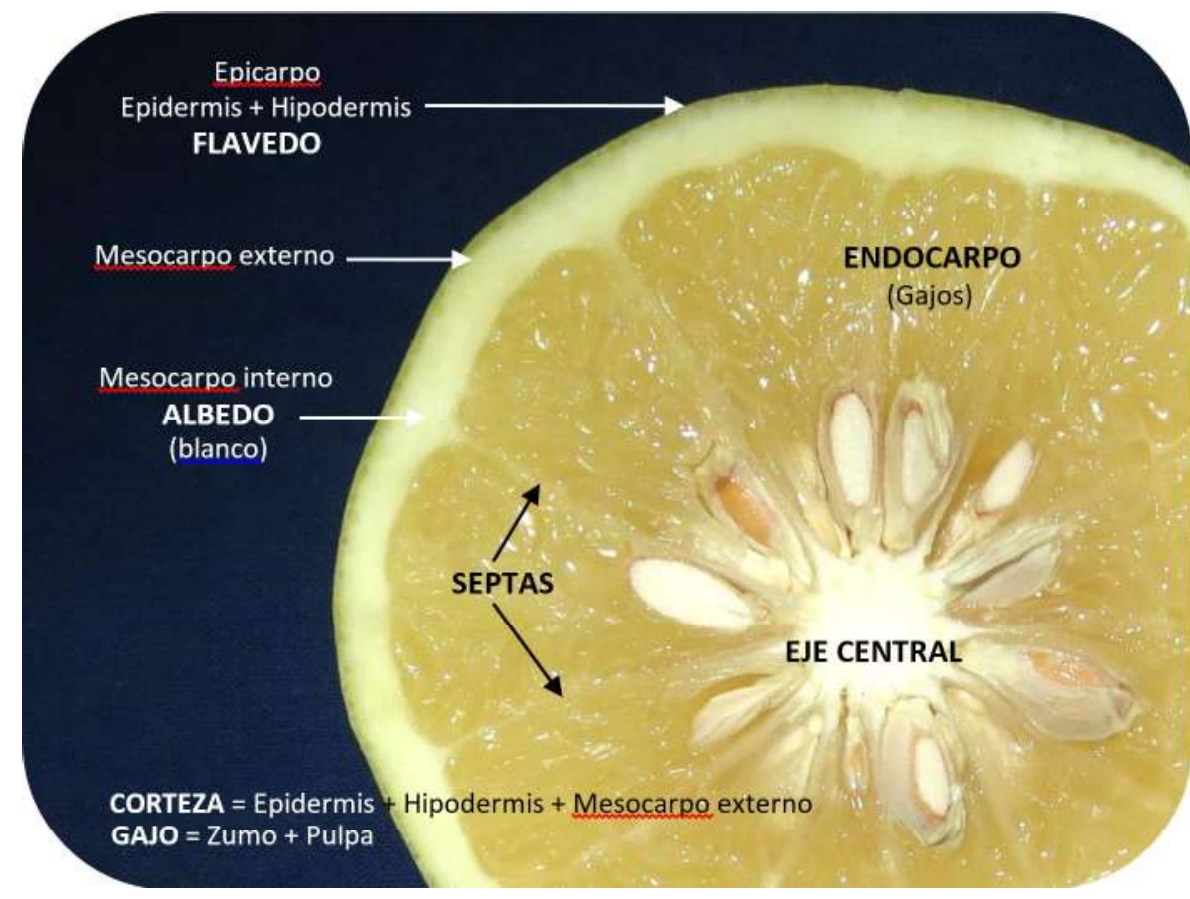

Figura 1. Partes de la toronja, siendo la corteza la parte utilizada para la obtención del aceite esencial.

estomatitis subprotésica (15).

Si bien es cierto que hay fármacos que tratan la estomatitis subprotesica, estos pueden generen en algunos casos, efectos adversos en su uso, resistencia fúngica, costo elevado para su aplicación, por lo que usar productos naturales como el Citrus paradisi que presenta en su composición D-limoneno, linalol o citral sustancias a la que se le atribuye acción antimicótica es una opción en la terapéutica para el tratamiento de la estomatitis subprotesica (16).

Por tanto, con el fin de tratar esta enfermedad a través de sustancias antifúngicas, se plantea la búsqueda de nuevos productos naturales que producirían menores efectos adversos que los fármacos tradicionales, además de estar al alcance de las clases más populares. El objetivo del presente trabajo fue determinar la actividad antifúngica del aceite esencial de Citrus paradisi "toronja" sobre cepas de Candida albicans.

\section{MATERIAL Y MÉTODOS}

\section{Recolección del material vegetal}

Se recolectaron y seleccionaron los frutos de toronja en estado de madurez, coloración amarilla y no lesionados.

\section{Extracción y dilución del aceite esencial}

Para la obtención del aceite esencial de toronja, los frutos fueron pelados manualmente utilizando un cuchillo afilado de acero inoxidable (17); las tiras finas obtenidas de su cáscara fueron sometidas a un proceso de hidrodestilación con arrastre de vapor de agua. El destilado del aceite esencial fue recibido en un embudo de decantación, aquí se observó un estado difásico entre agua y aceite esencial. Luego de decantado, el aceite esencial fue deshidratado utilizando sulfato de sodio anhidro (9). El aceite se depositó en un frasco de vidrio de color ámbar bajo refrigeración a temperatura de $4{ }^{\circ} \mathrm{C}(7)$.

El aceite esencial extraído fue diluido con dimetilsulfóxido (DMSO) obteniéndose concentraciones al 25; 12,$5 ; 6,25 ; 3,13 ; 1,56 ; 0,78$ y $0,39 \%$.

\section{Pacientes y muestras}

Se seleccionaron tres pacientes, para la toma de muestras según criterios de inclusión; como pacientes mayores de 18 años, edentulos parciales o totales con uso de prótesis removible dental, sistémicamente sanos, que no hayan recibido antimicrobianos antes de las dos semanas de la toma de muestra y con diagnóstico de estomatitis subprótesica en la cavidad oral. Los criterios de exclusión fueron; ser menores de 18 
años, pacientes que usan soluciones antimicrobianas o aquellos que no firmaron su consentimiento informado para la toma de muestra.

\section{Declaración de ética}

El estudio fue aprobado por el Instituto de Investigación Estomatológica de la Facultad de Odontología de la Universidad Nacional Mayor de San Marcos bajo lineamientos científicos y éticos. Todos los pacientes previa información detallada del estudio firmaban el consentimiento informado para la toma de muestra, la que no era nada invasiva, dolorosa o causara algún daño al paciente, sabiendo que dicha muestra será utilizada para fines de investigación.

Aislamiento e identificación de Candida albicans: Prueba de Tubo germinativo

Se realizó un frotis con hisopo estéril humedecido con suero fisiológico de la zona donde presentaban la lesión a cada uno de los pacientes elegidos. Las muestras fueron sembradas en agar Sabouraud e incubadas en condiciones de aerobiosis a $37^{\circ} \mathrm{C}$ por 48 horas. Posterior a la incubación, las colonias fueron reconocidas por sus características de crecimiento, como: circulares, convexas, elevadas, lisas, de un color blanco cremoso. A la microscopia presentaron forma típica de levaduras ovoides de 3 a $5 \mu \mathrm{m} \times 6$ a $12 \mu \mathrm{m}$. Una vez identificadas presuntivamente se resembraron en el mismo medio de cultivo para la obtención de las cepas puras y realizar la identificación. Para la identificación de Candida albicans por medio de la prueba del Tubo germinativo, se tomó una de las colonias características de cada cepa pura para ser suspendida en $0,5 \mathrm{~mL}$ de suero humano y se incubó a $35^{\circ} \mathrm{C}$ por 2 horas .Posteriormente una gota de la emulsión fue observada al microscopio, la prueba confirma la presencia de Candida albicans al visualizar la formación del tubo germinal (Figura 2), que es una estructura elongada que se origina a partir de la levadura (18-20).

\section{Determinación de la actividad antifúngica (Méto- do de difusión en agar)}

Esta prueba se basa en la inhibición del crecimiento fúngico, mediante la difusión de las sustancias activas en un medio sólido la que se evidencia con la formación de halos de inhibición (21).
Se prepararon los inóculos tomando una asada de las colonias de Candida albicans para ser suspendida en 5 $\mathrm{mL}$ de solución salina $0,9 \%$ hasta llegar a una concentración equivalente al grado de turbidez del tubo $\mathrm{N}^{\circ}$ 0,5 de la escala de McFarland (1-1,5 x $\left.10^{8} \mathrm{UFC} / \mathrm{mL}\right)$.

Se agregó $100 \mu 1$ de la muestra estandarizada a cada una de las 12 placas con agar Sabouraud, se hizo la siembra por diseminación y se dejó de 3-5 minutos para su secado. Con un sacabocado de $6 \mathrm{~mm}$ de diámetro se hicieron pozos de difusión para ser depositados $50 \mu \mathrm{L}$ de cada dilución del aceite esencial al $25 ; 12,5 ; 6,25 ; 3,13 ; 1,56 ; 0,78 \%$, control positivo (Clorhexidina $0,12 \%$ ) y control negativo (Dimetilsulfóxido) y se dejó en reposo por una hora para una mejor difusión de la muestra en el agar. Posteriormente, las placas fueron llevadas a incubación a 37 ${ }^{\circ} \mathrm{C}$ por 48 horas y con un vernier se tomó la medida de los diámetros de los halos de inhibición formados (Figura 3).

Tanto para la identificación fenotípica como para la prueba de sensibilidad, se utilizó como cepa patrón control, a la cepa de Candida albicans ATCC 10231, que sirvió para determinar su identificación así como su sensibilidad a Citrus paradisi. La prueba de sensibilidad es realizada siguiendo el protocolo similar al del CLSI (Clinical \& Laboratory Standards Institute) documento M44-P (22).

\section{RESULTADOS}

El aceite esencial de las cáscaras de Citrus paradi$s i$ "toronja" a las concentraciones de $25 ; 12,5 ; 6,25$; 3,13 y $1,56 \%$ demostraron actividad antifúngica sobre las cepas de Candida albicans con halos de inhibición promedio de 12,$63 ; 10,33 ; 7,79 ; 6,75$ y 6,29 $\mathrm{mm}$, estos resultados fueron llevados a la prueba estadística de Kruskal Wallis, determinando que existe diferencia estadística significativa $\mathrm{P} \leq 0,05$ entre los promedios de las concentraciones. Las concentraciones del aceite esencial al 0,78 y $0,39 \%$ no presentaron actividad antifúngica sobre las cepas de Candida albicans (Figura 4).

\section{DISCUSIÓN}

Nuestros resultados difieren de los reportados por Flores et al. (1999), que no encontró actividad antifúngica del aceite esencial de toronja al $1,0 \mathrm{mg} / \mathrm{mL}$ 


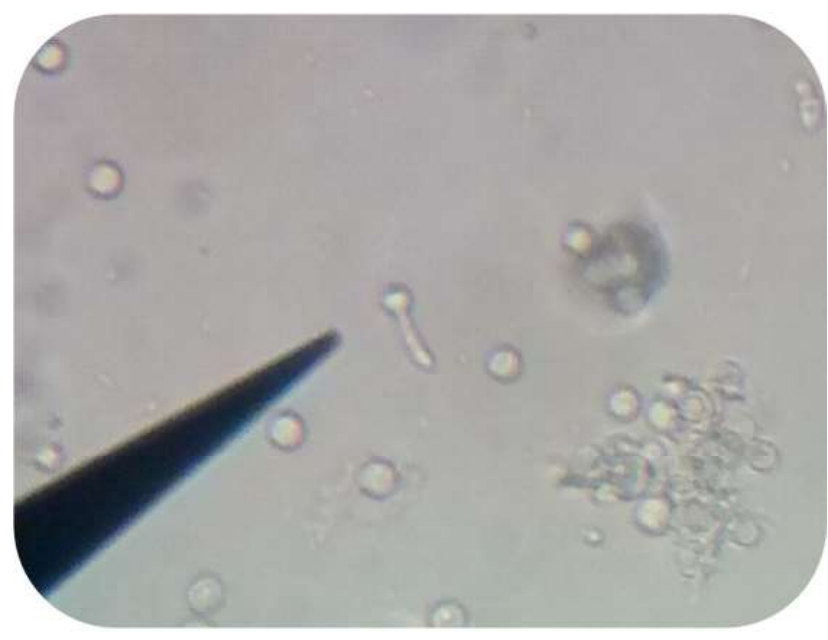

Figura 2. Prueba de tubo germinativo para identificación de Candida albicans, el puntero señala la extensión o tubo germinal que se forma luego de una incubación de la colonia de C. albicans en suero humano por un periodo de 2 horas.

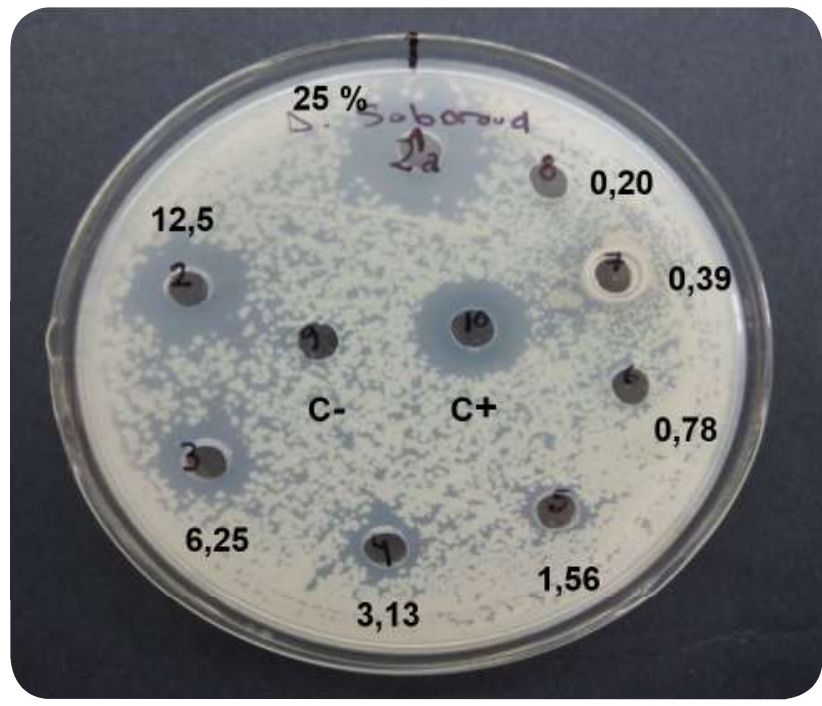

Figura 3. Test de sensibilidad en placa de agar con pozos, obsérvese que los diámetros de los halos de inhibición van decreciendo, conforme van disminuyendo las concentraciones y que el halo minino se observa para esta placa en $1,56 \% \mathrm{v} / \mathrm{v}$.

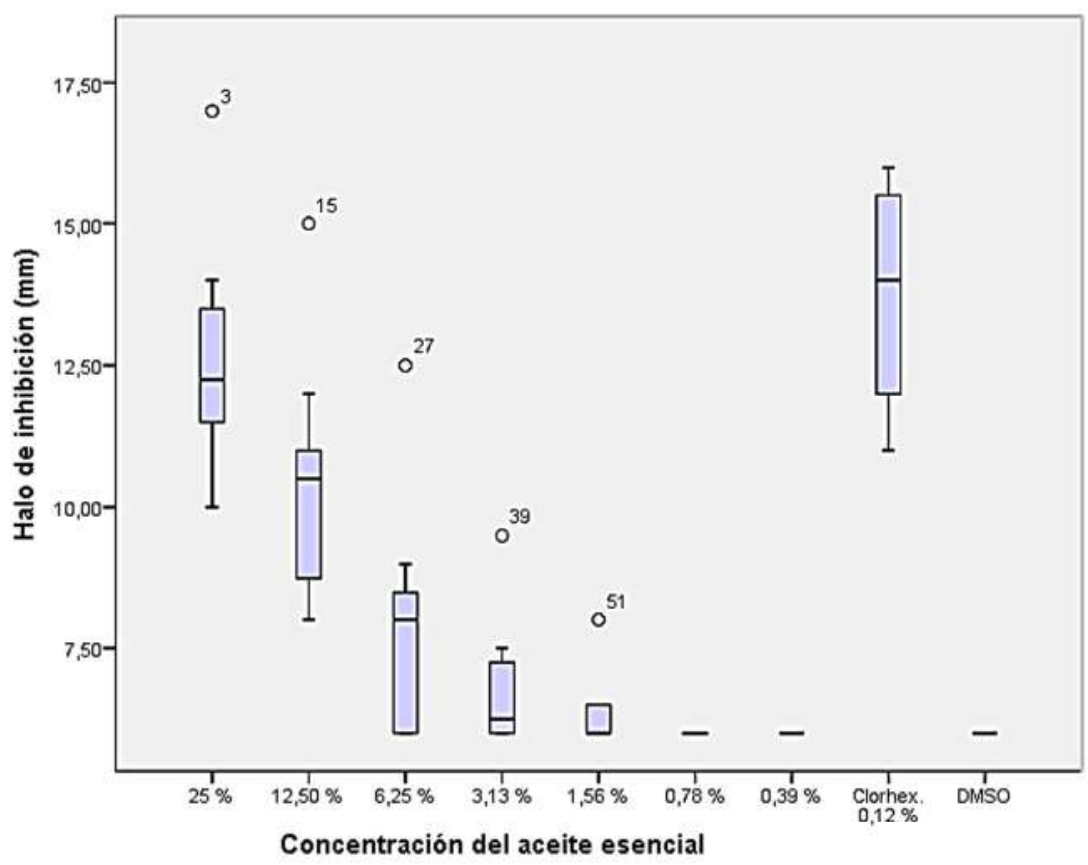

Figura 4. Diagrama de cajas en la que se relaciona las medianas según diferentes concentraciones del aceite y sus halos de inhibición. Obsérvese que concentraciones de $25 \%$ presenta una mediana de $12,25 \mathrm{~mm}$. Siendo esta de mayor valor que las demás concentraciones. 
sobre cepas de Candida albicans, pero si actividad antibacteriana contra Staphylococcus aureus, posiblemente la diferencia de estos resultados se deba al diluyente utilizado; Flores (8) utilizó el medio de cultivo para obtener las diferentes concentraciones, mientras en la presente investigación se utilizó el dimetilsulfóxido.

El comportamiento de Candida albicans, no mostró diferencias al comparar las concentraciones de aceite esencial entre 6,25 y $3,13 \%$, lo cual podría indicar que la concentración más baja $(3,13 \%)$ tendría el mismo efecto inhibitorio que la concentración al $6,25 \%$. Similarmente ocurre en las concentraciones de $3,13 \%$ y $1,56 \%$, en la cual una concentración de $3,13 \%$ sería igualmente efectiva que la de $1,56 \%$.

Según la escala de medición de Duraffourd, se obtuvo que el aceite esencial de Citrus paradisi al 25 y 12,5 $\%$ presentaron una actividad antifúngica límite y el aceite esencial al 6,$25 ; 3,13 ; 1,56 ; 0,78$ y $0,39 \%$ tuvo una actividad antifúngica nula (23). Estos resultados son similares a los obtenidos por Uysal (9) que determinó una actividad antibacteriana límite a sumamente sensible del aceite esencial de toronja contra Staphylococcus aureus, Enterococcus faecalis, Staphylococcus epidermidis, Escherichia coli, Salmonella typhimurium, Serratia marcescens y Proteus vulgaris,

La concentración mínima inhibitoria (CMI) promedio del aceite esencial de Citrus paradisi "toronja" frente a Candida albicans fue de 6,25\%.v/v. Este hallazgo no se puede comparar con otros estudios pues es un trabajo pionero en la búsqueda de la CMI del aceite esencial de la cáscara de Citrus paradisi. Sin embargo, estudios similares como el de Cvetnic (24) que determinó la CMI del extracto etanólico de semilla y pulpa de toronja, siendo el valor de CMI para $C$. albicans $8,25 \% \mathrm{~m} / \mathrm{v}$, el cual fue superior al obtenido en el presente trabajo con un valor de $6,25 \% \mathrm{v} / \mathrm{v}$.

\section{CONCLUSIONES}

Del estudio se concluye que el aceite esencial de $C i$ trus paradisi "toronja" presenta actividad antifúngica sobre cepas clínicas de Candida albicans, siendo la concentración al $25 \%$, la que presenta halos de inhibición de mayor diámetro, con una media de 12,63 $\mathrm{mm}$. Esta es una investigación pionera en la búsque- da de la CMI del aceite esencial de la cáscara de Citrus paradisi, así también viendo los beneficios antifúngicos de este producto natural se propone su posible uso para la estomatitis subprotésica, para lo cual, se tendría que realizar estudios complementarios.

\section{Agradecimientos}

Al Vicerrectorado de investigación de la Universidad Nacional Mayor de San Marcos, por el financiamiento del estudio así como al Instituto de Investigación Estomatológica de la Facultad de Odontología de la UNMSM, por la facilidad en el manejo operativo de la investigación.

\section{Correspondencia:}

Donald Ramos Perfecto

Correo electrónico:dramos_37@hotmail.com

\section{REFERENCIAS BIBLIOGRÁFICAS}

1. Araujo DJ, Salas AR. Actividad antimicrobiana de plantas. Científica. 2008; 6(2): 6-18.

2. Wen L, Haddad M, Fernandez I, et al. Actividad antifúngica de cuatro plantas usadas en la medicina tradicional peruana. aislamiento de 3' - formil - 2', 4',6' - Trihidroxidihidrochalcona, principio activo de Psidium acutangulum. Rev Soc Quím Perú. 2011; 77(3): 199-204.

3. Benavides V, D'Arrigo G, Pino J. Effects of aqueous extract of Origanum vulgare L. (Lamiaceae) on the preimplantational mouse embryos. Rev Peru Biol. 2010. 17(3): 381-384.

4. Macías WG. Proceso de obtención de extracto a partir de la semilla de toronja ( Citrus paradisi) y su aplicación en desinfección de vegetales o frutos y superficies planas. Tesis de Bachiller. Guayaquil: Facultad de Ingeniería Química, Universidad de Guayaquil; 2014.

5. Martínez CJ, Gutiérrez DM, Molina EZ, Rodríguez JD. Fertilización en cítricos en el estado de Nueva León. (Citado el 10 de mayo del 2015)Disponible en: http://www.agronomia.uanl.mx/

6. Gupta V, Kohli K, Ghaiye P, Bansal P. Pharmacological potentials of Citrus paradise: An overview. Int J Phytother Res. 2011; 1(1): 8-17.

7. Okunowo W, Oyedeji O, Afolabi L, Matanmi E. Essential oil of grape fruit (Citrus paradisi) peels and its antimicrobial activities. AJPS. 2013; 4: 1-9.

8. Flores E, Velasco P, Figueroa N, Gimenez A. Aceites esenciales con propiedades antimicrobianas. Biofarbo. 1999; 7: 5-8.

9. Uysal B, Sozmen F, Aktas O. Essential oil composi- 
tion and antibacterial activity of the grapefruit (Citrus Paradisi. L) peel essential oils obtained by solventfree microwave extraction: comparison with hydrodistillation. Int J Food Sci Tech. 2011; 46(7): 1455-1461.

10. Morales J, Gómez N, Rovira J, Abrahams M. Actividad larvicida de la toronja, Citrus paradisi (Rutaceae) sobre dos vectores del dengue. Rev Peru Biol. 2007; 14(2): 297-299.

11. Jawetz E, Melnick J, Adelberg E. Medical Microbiology. 25ava edición. Estados Unidos: Editorial Mc Graw Hill Lange, 2014.p. 159.

12. Gendreau L, Loewy Z. Epidemiology and etiology of denture stomatitis. J Prosthodont. 2011; 20(4): 251260.

13. Pardi G, Guilarte C, Cardozo E. Detección de Candida albicans en pacientes con candidiasis pseudomembranosa. Rev Odontol Univ Cid São Paulo. 2008; 20(3): 228-236.

14. Francisco A, Gonzalez Y, Sexto N, Vazquez A. Estomatitis subprotesis en pacientes portadores de protesis dental superior. MediSur. 2009; 7(1): 23-27.

15. Bruch J, Triester N. Clinical oral medicine and pathology. 1 ra edición. New York: Editorial Humana Press 2010.p. 92.

16. Viuda M, Ruiz Y, Fernandez J, Perez J. Antifungal activity of lemon ( Citrus lemon L.), mandarin (Citrus reticulate L.) grapefruit ( Citrus paradise L.) and orange ( Citrus sinensis L.) essential oils. Food control. 2008; 19:1130-1138.

17. Soto L. Composición y actividad microbiana del acei- te esencial de toronja (Citrus paradisi). Tesis de Magíster.Maracaibo, Venezuela: Facultad de Ingeniería, Universidad del Zulia; 2010.

18. Pemán J, Martín E, Rubio M. Guía Práctica de Identificación y Diagnóstico en Micología Clínica. Rev Iberoam Micol. 2007; 2(11):1-20.

19. Bonifaz A. Microbiología Médica Básica. $3^{\circ}$ edición. Ciudad de México: Editorial Mc Graw Hill; 2009.p. 8-30.

20. Linares M, Solís F. Guía práctica de identificación y diagnóstico en micología clínica-identificación de levaduras. Rev Iberoam Micol. 2007; 2(11):1-18.

21. Kalemba D, Kunicka A. Antibacterial and antifungal properties of essential oils. Curr Med Chem. 2003; 10(10): 813-829.

22. National Committee for Clinical Laboratory Standards. Methods for antifungal disk diffusion susceptibility testing of yeasts: approved standard M44-A. Wayne, USA: National Committee for Clinical Laboratory Standards; 2004.

23. 23.Duraffourd C, D'Hervicourt D, Lapraz J. Cuadernos de fitoterapia clínica. 1ra edición. Barcelona: Editorial Masson SA; 1986.p. 86.

24. 24.Cvetnic Z, Vladimir K. Antimicrobial activity of grapefruit seed and pulp ethanolic extract. Acta Pharm. 2004; 54(3): 243-250.

Recibido : 11/04/2016

Aceptado: 03/07/2016 